\title{
Geometric Damage Tensor Based on
}

Microplane Model

By Ignacio Carol,' Associate Member, ASCE, Zdeněk P. Bažant, ${ }^{2}$ Fellow, ASCE, and Pere C. Prat, ${ }^{3}$ Associate Member, ASCE

A8stRact: An appealing approach to formulate constitutive models for characterizing distributed damage due to microcracks and voids is continuum damage mechanics with the concepts of effective stress and strain equivalence. In that
approach. in which damage is imagined to characterize the reduction of the net approach, in which damage is imagined to characterize the reduction of the ne arated into two independent parts, onc for damage and the other for elastic and inelastic behavior (rheology) other than damage, which, if combined appropriately. give the overall constitutive behavior. However, the existing multidimensional formulations for damage are quite complex, and practical implementations capable of fitting experimental data are hard to obtain. The microplane models, by contrast. provide conceptual simplicity and close fits of multiaxial test data for concrete. soils, etc., although, as formulated in the past, various kinds of physical phenomen. were mixed in the definition of the microplane stress-strain curves. In this work the microplane theory is reformulated in a manner that scparates damage from rheology and makes the formulation fit the basic framework of continutum damage mechanics. Aside from a kinematic constraint between macrostrains and microstrains, the model satisfies a static constraint such that the effective microstresses are the resolved components of the effectiveness macrostresses.

\section{INTRODUCTION}

Inelastic phenomena are of two kinds: (1) Those that reduce material stiffness and cause strain-softening; and (2) those that do not. The former represent damage caused by nucleation, growth, and coalescence of microcracks and voids, and the latter consist of plastic slip, friction, creep, etc. Modeling each of these phenomena is difficult enough, but the complexity becomes formidable when both kinds of phenomena are combined, which occurs in many materials. In this paper we seek to separate these two phenomena to achieve simplification and clarity. We assume the microcracks and voids to remain homogeneously distributed so that the material can be microscopically treated as a continuum.

The real stress and strain fields at the microlevel are highly scattered and nonsmooth. Therefore, the continuum strains and stresses must be taken as the "average" values of these fields over a certain representative volume. However, applicability of damage in terms of such stresses and strains must be restricted to situations in which no localization phenomena take place, i.e., no macrocracking or shear-banding occurs. If the damage and strains

'Assoc. Prof of Civ Engrg., ETSECCPB-Tech. Univ, of Catalonia, 08034 Barcelona, Spain; formerly Visiting Scholar, Ctr. for Advanced Cement-Based Mat. Northwestern Univ Evanston, It 60208 .

${ }^{2}$ Walter P. Murphy Prof. of Civ. Engrg. , Ctr. for Advanced Cement-Based Mat. Northwestern Univ., Evanston, IL 60208

${ }^{3}$ Assoc. Prof. of Civ. Engrg., ETSECCPB-Tech. Univ. of Catalonia, $08034 \mathrm{Bar}-$ celona, Spain; formerly Visiting Scholar, Ctr. for Advanced Cement-Based Mat. Northwestern Univ., Evanston, IL.

Note. Discussion open until March 1, 1992. To extend the closing date one month a written request must be filed with the ASCE Manager of Journals. The manuscript for this paper was submitted for review and possible publication on Sept 1990 . This paper is part of the Journ of 1990. This paper is pat of Jo October, 1991. CASCE, ISSN 0733-9399/91/0010-2429/\$1.00+\$.15 per page. Pape No. 26299. 
can localize, unrealistic results can be obtained with this approach unless additional techniques such as crack band model, nonlocal models, or localization analysis are implemented in the context of the "smeared" approach to damage (Bažant and Cedolin 1979; Crisficld 1982; Pijaudier-Cabot and Bažant 1987; de Borst 1987; Ortiz 1987; Ortiz and Quigley 1989; Caro and Prat 1990). Alternatively, formulations can also be obtained by "discrete" crack models based on fracture mechanics with softening cohesive (crack-bridging) zone (Hillerborg et al. 1976; Hillerborg 1984).

The classical approach to distributed damage is the continuum damage mechanics. As originated by Kachanov (1958) for one-dimensional problems, the basic concept of continuum damage mechanics looks simple: a reduction of the net stress-carrying cross-section area fraction caused by the growth of microcracks and voids in the material microstructure. This is a purely geometric characteristic, reflecting the fact that the "effective" (or "true") stresses in the undamaged part of the cross section are higher than the stresses in the macroscopic continuum (the apparent stresses). A law can be postulated to predict the decrements of the current cross-sectional area in terms of stress, strain, and damage, and simple but useful results can be obtained for complex phenomena such as creep rupture of metals (Kachanov 1958; Rabotnov 1969a,b; Leckie 1978; Lemaitre 1984) and, more recently, the delayed failure of concrete under sustained uniaxial compression (Carol 1989).

The multiaxial generalization of continuum damage mechanics, however poses a formidable challenge that, to a large extent remains unsolved. The net stress-carrying area fractions for the different directions in space cannot be easily integrated to give a vectorial or tensorial damage measure that would define the "effective" or "true" stress tensor from the macroscopic stress tensor and the apparent or reduced macroscopic stiffness tensor. A review of the existing multiaxial generalizations presented in the section headed "Existing Models for Distributed Damage" shows a number of possibilities, some of them rather complicated and not always very satisfactory in terms of data-fitting capabilities, especially when the classical macroscopic tensorial approach is pursued. This is the case when the constitutive model is formulated phenomenologically without recourse to any geometric concepts related to microstructure and the inelastic phenomena are formulated as functions of stress and strain invariants. Nevertheless, as will be shown in the section headed "Effective Stress and Damage Tensor Uncoupled from Rheology" some basic general structure for continuum damage models based on the concepts of true, or effective, stress and macromicro strain compatibility can also be established in three dimensions. This basic structure provides a suitable framework for separating the constitutive model in two parts, one for damage and the other for rheology, each of which can be formulated independently and then combined. This provides a convenient flexibility for combining damage with various complex types of inelastic material behavior.

Compared to multiaxial continuum damage model theories, much closer fits of test data for concrete and considerable theoretical simplification (albeit at the expense of computer time requirements) is achieved by the microplane models. In general terms, already suggested for plasticity in a famous classical paper by G. I. Taylor (1938), this approach characterizes the material properties independently on planes of various orientation, which were initially called the slip planes (Batdorf and Budiansky 1949) but renamed microplanes when the concept was extended to materials incapable of plastic slip (Bažant 1984). The basic material behavior is defined on the microplanes as a set of relations hetween the stresses and strains acting on that plane. These relations include all the rheologic phenomena involved in material behavior (elasticity, damage, plasticity) at the same time, in an inseparable manner. The microplane behavior is constrained to the macroscopic continuum behavior by a suitable macro-micro constraint having the form of a variational equation based for example on the principle of virtual work. In some studies (Bažant 1984; Bažant and Oh 1983, 1985; Bažant and Gambarova 1984; Bažant and Prat 1988; Carol et al. 1990) the microplane concept, originally developed for metal plasticity, was extended to materials exhibiting damage with strain softening. The resulting model, briefly described in the section headed "Kinematically Constrained Microplane Model," was shown to be capable of a very good description of the existing test data on tensile strain-softening of concrete due to microcracking as well as the nonlinear triaxial behavior of concrete, including strain-softening in compression and shear under different confining lateral stresses.

This paper [which was briefly summarized in a conference paper by Bažant and Carol (1990)] reconciles both types of constitutive models (continuum damage and microplane), and presents a new interpretation of the microplane formulation as a continuum damage model. This leads to the formulation of a fourth-order nonsymmetric geometric damage tensor. The term "geometric" reflects the fact that this tensor is independent of any particular rheologic model, and depends only on the reduction of the net stress-carrying area fraction in various directions in the material, which are geometric characteristics (the term "geometric," of course, does not imply that all the geometric characteristics, such as spatial crack and void configuration and statistical distributions, are taken into account). The proposed geometric damage tensor achieves a three-dimensional generalization of Kachanov's (1958) one-dimensional net stress-carrying area fraction. It is computed by integrating the geometric damage at each particular microplane over all the spatial directions. As a continuum damage model, it gives the relationship between the macroscopic and effective stress tensors and between the initial and current stiffness matrices, and can be combined with any rheologic model (elasticity, plasticity, viscoelasticity). As a microplane formulation, it also has the additional advantage of an a priori knowledge that very good data fitting can be obtained in practical applications. The derivation of the new damage tensor is presented in two different ways: In the section headed "Derivation of Geometric Damage Tensor from Concrete Model" it is derived from a version of the microplane model with particular microplane laws used previously for concrete (Carol et al. 1990), and in the section headed "Rheology-Independent Derivation of Geometric Damage Tensor" it is derived without recourse to any particular stress-strain laws.

\section{Existing Models for Distributed Damage}

Consider first the example of a simple one-dimensional continuum damage formulation. In this case damage such as microcracks or voids may be regarded as geometric, representing a reduction in some ratio $\alpha$ of the net stress-carrying area in the microstructure of the material [Fig. 1(a)]. For uniaxial stress (macrostress) o, we may thus write

$\sigma=\alpha \tau$ 


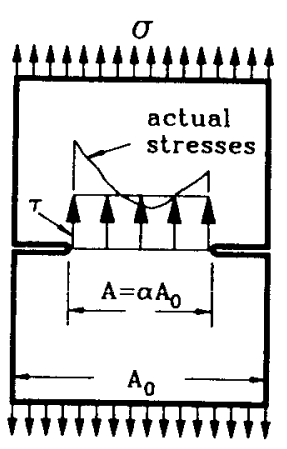

(a)

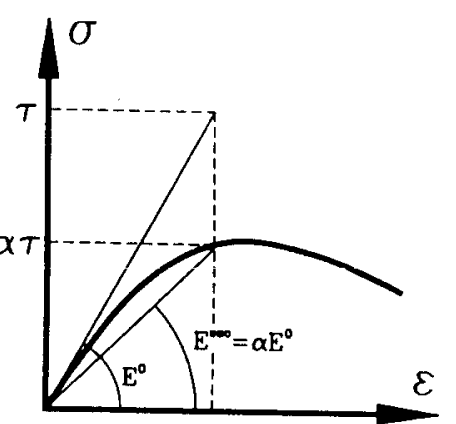

(b)
FIG. 1. Effective Stress and Damage in One Dimension: (a) Interpretation of $\tau$ and $\alpha$ (b) Stress-strain Diagram

in which $\tau$ is called the effective (or true) stress; and $\alpha$ varies from 1 to 0 when damage increases (alternatively, damage can also be represented by $\omega=1-\alpha$ varying from 0 to 1$) ; \tau$ represents the average stress in the stress-carrying portion of the cross-sectional area in the microstructure. Assuming a linear elastic relation, i.e., $\tau=E^{0} \varepsilon$, where $E_{0}$ is the elastic modulus of the undamaged material and $\varepsilon$ the strain, one has

$\boldsymbol{\sigma}=\alpha E^{0} \varepsilon$

This equation may also be written as

$\boldsymbol{\sigma}=E^{\sec } \boldsymbol{\varepsilon}$

$E^{\mathrm{sec}}=\alpha E^{\prime}$

where $E^{\mathrm{sec}}=$ secant modulus [Fig. $1(b)$ ]. Now, a damage evolution law can be assumed for $\alpha$. If it is defined in terms of $\varepsilon$ and introduced in (2) or (3), an explicit one-dimensional stress-strain relation for damage ensues. As seen in this simple example, the formulation of a continuum damage model includes three steps.

1. Characterization of damage by means of a variable ( $\alpha$ or $\omega$ ) or set of variables.

2. Dependence of the effective stress or secant modulus on the damage variable $\left(\tau=\sigma / \alpha\right.$ or $\left.E^{\mathrm{sec}}=\alpha E^{\prime}\right)$

3. Evolution laws for the damage variable; e.g. $\dot{\alpha}=F(\sigma, \dot{\varepsilon}, \alpha \cdots(F=$ function or functional).

In triaxial generalization, some early models included damage-related concepts (e.g. stiffness degradation) that did not explicitly introduce a damage variable as such, but instead derived the evolution laws from a "fracturing surface" with attributes similar to the classical yield surface in plasticity, defining this surface in terms of stress and strain invariants with hardening-softening rules, internal variables, etc. (Dougill 1976; Bažant and Kim 1979). That was a phenomenologic approach to the problem whose most sophisticated forms became impractical because of the high complexity and the presence of too many parameters and empirical functions, giving little insight into the physical processes actually occurring at the microstructural level.

Most of the existing theories dealing with three-dimensional stiffness degradation incorporate some kind of damage variable in their definition and can be satisfactorily described in terms of the foregoing three-step scheme. The simplest possible characterization of three-dimensional damage is a scalar variable, which can be thought of as the damage caused by a field of spherical microvoids with the same stress-carrying area reduction in all spatial directions (Leckie 1978; Lemaitre and Chaboche 1978; Mazars 1985; Resende 1987; Frantziskonis and Desai 1987). To capture the anisotropic nature of damage caused by microcracks with predominant orientation, damage tensors of various orders have been proposed: vectors (Davidson and Stevens 1973; Krajcinovic and Fonseka 1981; Suaris and Shah 1984; Costin 1985; Costin and Stone 1987); second-order tensors (Vakulenko and Kachanov 1971; Dragon and Mróz 1979; Kachanov 1980; Murakami and Ohno 1980; Betten 1983; Oda 1983; Murakami 1987: Suaris 1987); fourthorder tensors (Chaboche 1979, 1981; Ortiz 1985; Simó et al. 1987; Yazdani and Schreyer 1988), or even eighth-order tensors (Chaboche 1978).

The influence of the damage variable on the secant stiffness or effective stresses, along with the definition of the laws for damage evolution (steps 2 and 3), depend much on what kind of damage variable is adopted. Using a scalar damage variable, these assumptions can be made analogous to the one-dimensional case, but then no induced anisotropy can be accounted for. Some authors use the secant stiffness itself (or a related quantity such as the increment of compliance over the initial or elastic one) as a fourthorder damage tensor (Ortiz 1985; Simó et al. 1987; Yazdani and Schreyer 1988). With this approach, the dependence in step 2 is defined implicitly, but the stiffness tensor does not have a direct physical meaning as a damage variable, and the corresponding evolution laws (step 3) have to be established again, mainly on a phenomenologic basis.

In a certain sense the intermediate option of a second-order tensor as a damage variable seems appealing in the face of other possibilities. Consider for instance the "fabric tensor" proposed by Oda (1983) for the analysis of cracked rock masses, which was an improvement over other previous similar proposals. This tensor is defined as an integral over all the possible crack orientations and sizes of an expression including the crack density function. It is a symmetric dimensionless tensor, and some of its invariants can be interpreted in terms of the overall geometric effect caused by the field of microcracks. The concept of a second-order damage tensor also seems to be supported by theoretical considerations relative to frame invariance (Leckie and Onat 198(), as well as by experimental microstructural measurements of the corresponding "damage ellipsoid" in quite different materials (e.g. measurements of cancellous bone structure and granular soil fabric; Cowin 1985). Unfortunately, the attractive concept of a second-order fabric tensor contrasts with the absence of equally clear and direct relations for the degradation of the macroscopic secant stiffness tensor and for the effective stresses in the cracked medium. Cowin (1985) deduces the most general dependence of the secant stiffness on the second-order fabric tensor permitted by the condition of frame invariance, which turns out to be a complex polynomial expression, with the somewhat disappointing peculiarity that at least a quadratic (and likely even higher) dependence on the first invariant 
of the fabric tensor must be involved in the relation if orthotropic symmetry is to be preserved in the cracked material. Some other authors, on the other hand, handle the problem of reduction of the net stress-carrying area due to microcracks through simulation of a finite deformation field with the equivalent effects of area reduction in various directions in space, or through certain geometric and tensorial considerations. However, either they do not use a second-order tensor as a damage variable [Krajcinovic and Fonseka (1981), who used a vector], or they get an awkward nonsymmetric effective stress tensor, which must further be symmetrized in some arbitrary way in order to obtain a practical formulation (Murakami and Ohno 1980; Betten 1983)

A different approach is suggested by studies of a homogeneous medium containing periodic arrays of inclusions or penny-shaped cracks. In such studies, the overall elastic modulus is obtained by calculating the approximate stress fields in the solid, by the so-called self-consistent method or other related techniques (Budiansky and O'Connell 1976; Nemat-Nasser et al. 1982; Horii and Nemat-Nasser 1983; Attiogbe and Darwin 1986; Kachanov 1987; Aboudi 1987). This approach can be useful when the overall modulus of a continuum (e.g. rock, a composite material) containing an a priori known field of cracks or inclusions needs to be computed. However, in most of those theories the field of microcracks and the configuration of inclusions must be known in some detail; in constitutive modeling a damage variable characterizes these effects in a smeared way. Also, the relationships obtained for step 2 are usually very complicated (and sometimes they even do not have an explicit form but are a set of nonlinear equations to be solved numerically for each practical case); they should be explicitly tractable expressions to be introduced as one component of the constitutive model. As a result [although some authors did use this approach to derive or support the choice of relations included in step 2; (Chaboche 1979; Oda 1983)], this approach in general seems to be only of limited usefulness for the development of complete macroscopic constitutive relationships.

\section{Effective Stress and Damage Tensor Uncoupled from}

\section{RHEOLOGY}

To define the effective stress in three dimensions the following tensorial expression similar to (1) for one dimension has been introduced (Rabotnov 1969a,b; Chaboche 1979; Lemaitre and Chaboche 1985; Simó et al. 1987):

$\sigma_{i j}=\alpha_{i j k m} \tau_{k m}$

where the repetition of lower-case indices implies summation over 1, 2, 3; $\sigma_{i j}$ and $\tau_{k m}=$ Cartesian components of the macroscopic and effective (or true) stress tensors $\sigma$ and $\tau$; and $\alpha_{i j k m}=$ components of a fourth-order dimensionless tensor $\alpha$ characterizing damage (which can also be expressed as $\alpha=I-\omega$, where $I=$ fourth-order identity tensor; and $\omega=$ alternative damage tensor)

Symmetry is assumed for the macroscopic stresses $u$ as well as for the effective stress tensor $\tau$. Thus, the components $\alpha_{i j k m}$ must preserve the interchangeability of index $i$ with $j$ and $k$ and $m$, and can be grouped into a $6 \times 6$ matrix in the context of the six-component vectorial representation of symmetric stress tensors. However, no interchangeability of indices $i, j$ with $k, m$ is assumed, which means that no symmetry is required for the
$6 \times 6$ matrix. This nonsymmetry is needed so as not to exclude the possibility of representing phenomena such as internal friction.

To establish more precisely the meaning and nature of the damage tensor $\alpha$ and effective stresses $\tau$, the hypothesis of strain equivalence is also assumed (Chaboche 1979; Lemaitre and Chaboche 1985; Simó et al. 1987). According to this hypothesis, the effective stresses $\tau$ are defined as the stresses that would exist in the undamaged material subjected to the same strains $\varepsilon$ as those that produce the macroscopic stress $\sigma$ in the damaged material. With this definition the components of the $6 \times 6$ damage tensor $\alpha$ can be geometrically interpreted as a net (effective) stress-carrying area fraction in every coordinate direction, with each stress component considered to act independently on a plane of that direction.

In the relationship between $\tau$ and $\varepsilon$, any rheologic model can be used; e.g., using linear elasticity $\left(\tau_{k m}=D_{k m p q}^{\mathrm{el}} \varepsilon_{p q}\right)$ as the rheologic model, one immediately obtains the expression for the secant or reduced elastic stiffness

$\sigma_{i j}=D_{i j p q}^{\mathrm{sec}} \varepsilon_{p q}$

$D_{i j k m}^{\mathrm{sec}}=\alpha_{i j r s} D_{r s p q}^{\mathrm{el}}$

These equations are the three-dimensional counterpart of (3).

In general, a nonlinear rheologic model may be used instead of linear elasticity to compute the effective stresses $\tau$ from prescribed strain $\varepsilon$ (which can in general be done according to a step-by-step algorithm). Because the value of $\alpha$ can be computed independently according to the damage laws the macroscopic stress $\sigma$ can then be obtained using (4). Thus, the overall constitutive model can be decomposed in two independent parts, one for damage and another for rheology. Different models can be used for each part and then combined. Establishing the laws for the evolution of $\alpha$, one can get an entire new class of models from the combination of the $\alpha$ evolution law with any rheologic model for $\tau$. This opens new interesting possibilities and presents the challenge of developing a satisfactory law for the evolution of the damage tensor $\alpha$, probably the weakest point at present and the main goal of this paper.

It should be pointed out that the idea of combining damage and rheology is not new, of course. For example Leckie (1978) and Lemaitre and Chaboche (1978) combined viscoplasticity and damage, and Bažant and Chern (1985) combined concrete creep with damage due to smeared cracking. The new idea is to combine damage and rheology by means of the microplane model, which has the benefit of yielding the form of the damage tensor.

\section{Kinematically Constrained Michoplane Model}

As already mentioned, the new damage tensor proposed here was derived by reformulating the latest version of the microplane model for concrete in an explicit form (Carol et al. 1990). Therefore, only the basic hypotheses and main features need to be given here.

The model is based on the concept of a microplane. A microplane is an arbitrary plane on which the constitutive properties are defined. Instead of defining them by means of a relation between tensors $\sigma_{i j}$ and $\varepsilon_{i j}$, these properties are defined by means of a relation between the stress and strain components on a microplane, which is conceptually much simpler because there are fewer stress and strain components and the problems of tensorial invariance do not arise on the microplane level. The macroscopic stress- 
strain relationship is then obtained by integrating under some suitable micromacro constraint over the microplanes of all spatial orientations $n$. This integration guarantees the condition of tensorial invariance to be satisfied automatically. There are two simple alternatives for the macro-micro constraint relating the behavior of all the microplanes to the macroscopic behavior: the static and kinematic constraints, both suggested by Taylor (1938). In the static constraint, the microplane stresses represent the projections of the macroscopic stress tensor on the plane considered; the kinematic constraint does the same for strains. Note that both types of constraints cannot apply at the same time if general stress-strain laws are considered at the microplane level.

Although the static constraint was used exclusively in the works dealing with metal plasticity (Batdorf and Budiansky 1949; Lin 1968), the kinematic constraint was recognized to be necessary to ensure that the microplane system be stable in strain-softening behavior (Bažant 1984; Bažant and $\mathrm{Oh}$ $1983,1985)$. If the kinematic constraint is assumed, the normal and shear strains $\varepsilon_{N}$ and $\varepsilon_{T_{i}}$ on the microplane with unit normal $n_{i}$ [Figs. $2(a$ and $b)$ ] can be obtained as projections of the strain tensor $\varepsilon_{i}$. Previous studies (Bažant and Prat 1988) revealed that in order to obtain any desired Poisson ratio $v(-1 \leq v \leq 0.5)$ as well as capture the effect of the hydrostatic pressure on the incremental stiffness, the normal microplane strain $\varepsilon_{N}$ needs to be further split into its volumetric and deviatoric components $\varepsilon_{v}$, and $\varepsilon_{f}$. This split, however, has no direct physical meaning; rather, the physical meaning is that the microplane stress depends not only on $\varepsilon_{N}$ but also on the lateral normal stress $\varepsilon_{L}$ along the directions lying within the microplane (the mean value of $\varepsilon_{L}$ can be easily expressed in terms of $\varepsilon_{V}$ and $\left.\varepsilon_{D}\right)$ ). The resulting expressions for the microplane strains are

$\varepsilon_{V}=\frac{\varepsilon_{k k}}{3}=\frac{\delta_{k m}}{3} \varepsilon_{k m}$

$\varepsilon_{D}=\left(n_{k} n_{m}-\frac{\delta_{k m}}{3}\right) \varepsilon_{k m}$

$\varepsilon_{T_{i}}=\frac{1}{2}\left(\delta_{i k} n_{m}+\delta_{i m} n_{k}-2 n_{i} n_{k} n_{m}\right) \varepsilon_{k}$
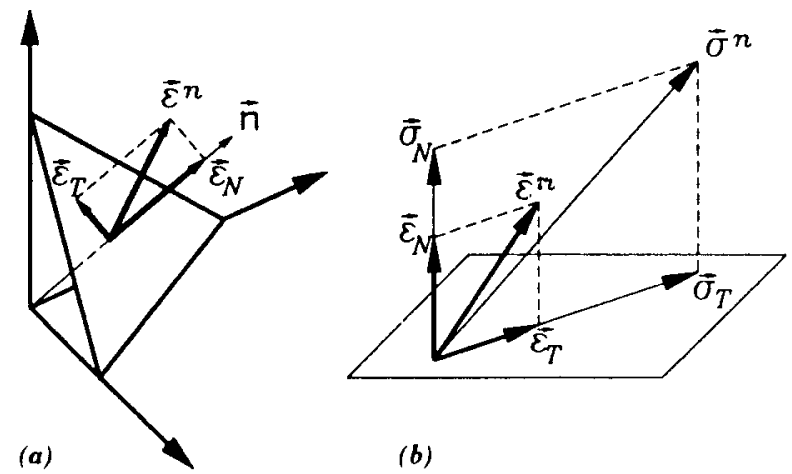

(b)

FIG. 2. Stress and Strain Components on Microplane where $\delta_{i j}=$ Kronecker delta; and $n_{i}=$ direction cosines of the unit vector $n$ normal to the microplane. Note that $\varepsilon_{D}$ and $\varepsilon_{T ;}$ can also be obtained as the normal and shear components of the projection of the deviatoric strain tensor $e_{i j}=\varepsilon_{i j}-\varepsilon_{\imath} \delta_{i j}$ on the microplane, but that the deviatoric part of the strain projection, which is $\varepsilon_{T_{i}}=\varepsilon_{i r} n_{j}-\varepsilon_{N} n_{i}$, differs from (6). Eq. (6) for $\varepsilon_{T_{i}}$ is obtained as the symmetric part of this tensor (the asymmetric part has no effect on stresses)

In view of the kinematic constraint imposed, the microplane stresses in general do not and cannot represent the resolved components of the (macroscopic) stress tensor. Equilibrium must nevertheless be imposed in an approximate overall sense. For this purpose, application of the principle of virtual work was proposed by Bažant (1984). This principle yields the following condition of static equivalence of the stress (macrostress) tensor $\sigma_{i j}$ with the normal $\left(\sigma_{N}=\sigma_{v}+\sigma_{D}\right)$ and shear stresses $\left(\sigma_{i}\right)$, on the microplanes:

$\frac{4 \pi}{3} \sigma_{i j} \delta \varepsilon_{i j}=2 \int_{\Omega}\left(\sigma_{N} \delta \varepsilon_{N}+\sigma_{T} \delta \varepsilon_{T}\right) f(n) \mathrm{d} \Omega$

The left-hand side represents the virtual work of the macrostresses in an elementary unit sphere; and the right-hand side represents the virtual work of the microplane stresses on the microplane strains on all the elementary facets on this sphere, representing the microplanes. The integration needs to be carried out only over the area of a unit hemisphere, $\Omega$, because the stresses and strains at its diametrically opposite points are equal. Function $f(n)$ may be used to introduce initial material anisotropy. For isotropic materials, $f(n) \equiv 1$

Substituting microplane strain variations according to (1) and (2), and setting $\sigma_{N}=\sigma_{v}+\sigma_{D}$, where $\sigma_{N}, \sigma_{v}$, and $\sigma_{D}$ are the normal, volumetric, and deviatoric stress components on the microplanes, we obtain

$\left\{\int_{\Omega}\left[\left(\sigma_{V}+\sigma_{I}\right) n_{i} n_{j}+\frac{\sigma_{T_{r}}}{2}\left(n_{i} \delta_{r j}+n_{j} \delta_{r i}-2 n_{i} n_{r} n_{r}\right)\right] f(n) \mathrm{d} \Omega\right.$

$\left.-\frac{2 \pi}{3} \sigma_{i j}\right\} \delta \varepsilon_{i j}=0$

This is a variational equation that must be true for any variation $\delta \varepsilon_{i j}$. This occurs if and only if the expression within braces, \{\} , vanishes. Noting also that $\int_{\Omega} n_{i} n_{j} \mathrm{~d} \Omega=2 \pi \delta_{i j}$, one obtains the expression

$\sigma_{i j}=\sigma_{V} \delta_{i j}+\int_{\Omega} \sigma_{D} n_{i} n_{j} \mathrm{~d} \Omega+\int_{\Omega} \frac{\sigma_{T_{r}}}{2}\left(n_{i} \delta_{r j}+n_{j} \delta_{r i}-2 n_{i} n_{j} n_{r}\right) \mathrm{d} \Omega \ldots$

Notice that, in fact, not all the components of $\delta \varepsilon_{i j}$ in (8) can have independent variations, since $\varepsilon$ is a symmetric tensor. In principle, this would complicate somewhat the elimination of this term from both sides of the equation (Carol et al. 1990). However, thanks to the symmetry (between $i$ and $j$ ) in the expressions used [(6)], the resulting expression between brackets, [ ], in (8) is also symmetric with respect to the interchange of indices $i$ and $j$, which makes this simplification possible (the same remark also applies to other similar simplifications of tensorial variables from other expressions with similar structure later in the paper).

The formulation needs to be supplemented by particular microplane stressstrain laws for $\sigma_{V}, \sigma_{D}$, and $\sigma_{T i}$ as functions of $\varepsilon_{V}, \varepsilon_{D}$, and $\varepsilon_{T}$. As shown by 
Bažant and Prat (1988), these can be assumed to be algebraic explicit relations (rather than differential equations) separate for each component. i.e. (for loading)

$\sigma_{V}=F_{v}\left(\varepsilon_{v}\right)$

$\sigma_{D}=F_{D}\left(\varepsilon_{D}\right)$

$\sigma_{T_{i}}=\frac{F_{T}(\gamma)}{\gamma} \varepsilon_{T}$

where $\gamma=\sqrt{\varepsilon_{T_{r}} \varepsilon_{T_{r}}}$ (the response, however, is in general path-dependent because various combinations of loading and unloading on various microplanes arise, even for macroscopically monotonic loading). A further simplifying hypothesis, that the shear stress $\sigma_{T_{i}}$ is parallel to the shear strain $\varepsilon_{r_{i}}$, is also made. In that case it suffices to specify the constitutive relation for the magnitude $\sigma_{T}=\sqrt{\sigma_{T}, \sigma_{T r}}$ rather than all the components $\sigma_{7}$. In previous works (Bažant and Prat 1988; Carol et al. 1990) the simplified relations in (10) did not prevent achieving good fits of a wide range of test data for concrete.

The computation of a prescribed-strain macroscopic load step can be performed explicitly: the microplane strain increments are computed from the macroscopic strain increment using (6). Then the new microplane stresses are obtained using the microplane laws, (10) (for each microplane one must know whether unloading started and, if so, which unloading path it is following). And, finally, the microplane stresses are integrated according to (9) to obtain the new macroscopic stresses.

\section{Derivation of Geometric Damage Tensor from Concrete Model}

The microplane constitutive laws from (10) can be rewritten in the form of elastic-damage laws with secant moduli $E_{V}^{\mathrm{sec}}, E_{D}^{\mathrm{sec}}$, and $E_{T}^{\mathrm{sec}}$

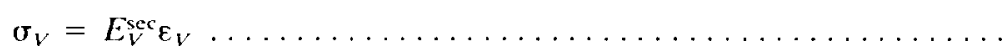

$\sigma_{D}=E_{D}^{\sec } \varepsilon_{D}$

$\sigma_{T_{i}}=E_{T}^{\mathrm{sec}} \varepsilon_{T_{i}}$

where $E_{V}^{\mathrm{sec}}=f_{V}\left(\varepsilon_{V}\right)$, etc. $\left(f_{V}=\right.$ function). Introducing (11) and the expressions of microplane strains from (6) in the right-hand side of (9), replacing the macroscopic stress in the left term by its elastic-damage expression with secant stiffness tensor from (5) for $\sigma_{i j}$, and eliminating $\varepsilon_{i j}$ from both sides of the resulting equation one obtains the macroscopic secant stiffness tensor expressed as an integral of the secant stiffness at microplane level over the unit hemisphere

$D_{k m p q}^{\mathrm{sec}}=\frac{E_{V}^{\mathrm{sec}}}{3} \delta_{k m} \delta_{p q}+\frac{3}{2 \pi} \int_{\Omega} E_{D}^{\mathrm{sec}} n_{k} n_{m}\left(n_{p} n_{q}-\frac{\delta_{p q}}{3}\right) \mathrm{d} \Omega$

$+\frac{3}{2 \pi} \int_{\Omega} \frac{E_{T}^{\mathrm{sec}}}{4}\left(n_{k} n_{p} \delta_{m q}+n_{k} n_{q} \delta_{m p}+n_{m} n_{p} \delta_{k q}\right.$

$\left.+n_{m} n_{q} \delta_{k p}-4 n_{k} n_{m} n_{p} n_{q}\right) \mathrm{d} \Omega$

Now the following substitutions may be introduced: (1) The secant stiffness at macroscopic level is replaced by the product of the initial elastic stiffness and the damage tensor $\left[(5)\right.$ for $\left.D_{i j p q}^{\text {ec }}\right]$ : (2) similar replacements are made for the microplane secant moduli:

$E_{V}^{\mathrm{sec}}=\alpha_{V} E_{V}^{0}$

$E_{i}^{\mathrm{sec}}=\alpha_{D} E_{D}^{\prime \prime}$

$E_{T}^{\mathrm{sec}}=\alpha_{T} E_{T}^{\mathrm{o}}$

where $\alpha_{v}, \alpha_{D}$, and $\alpha_{T}=$ microplane damage variables reflecting the net stress-carrying area fraction at microplane level; and $E_{V}^{0}, E_{D}^{0}$, and $E_{T}^{(1}=$ initial elastic moduli; (3) the initial moduli $E_{v}^{0}, E_{D}^{0}$, and $E_{7}^{0}$ are replaced by their expressions in terms of the macroscopic elastic parameters $E$ and $v$ and the additional parameter $\eta_{0}$ (Bažant and Prat 1988)

$E_{\nu}^{\prime \prime}=\frac{E}{1-2 \nu}$

$E_{D}^{\gamma}=\eta_{0} E_{V}^{\psi}$

$E_{T}^{\prime \prime}=\frac{1}{3}\left[\frac{5(1-2 v)}{1+v}-2 \eta_{0}\right] E_{v}^{\prime}$

and (4) the elastic stiffness matrix is replaced by its expression:

$D_{k m p q}^{c l}=\frac{v E}{(1+v)(1-2 v)} \delta_{k m} \delta_{p q}+\frac{E}{(1+v)} \delta_{k p} \delta_{m q} \ldots \ldots \ldots \ldots$

Once all these expressions are introduced into (12) and simplified, an expression for $\alpha_{i j k}$ is obtained in terms of $\alpha_{v}, \alpha_{l}$, and $\alpha_{T}$. Now an interesting idea comes to mind: parameters $E, v$, and $\eta_{1}$ can vanish from the expression for $\alpha_{i j k m}$ when the following assumption is made:

$\eta_{0}=\frac{1-2 v}{1+v}$

As we will see later, this hypothesis appears to be acceptable for practical purposes, and one gets

$\alpha_{i p q}=\frac{\alpha_{V}}{3} \delta_{i i} \delta_{p q}+\frac{3}{2 \pi} \int_{\Omega} \alpha_{D} n_{i} n_{j}\left(n_{p} n_{q}-\frac{\delta_{p q}}{3}\right) \mathrm{d} \Omega$

$+\frac{3}{2 \pi} \int_{\Omega} \frac{\alpha_{T}}{4}\left(n_{i} n_{p} \delta_{j q}+n_{i} n_{q} \delta_{m}+n_{i} n_{p} \delta_{i q}\right.$

$\left.+n_{j} n_{q} \delta_{i p}-4 n_{i} n_{j} n_{p} n_{q}\right) \mathrm{d} \Omega$

The fact that this tensor is purely geometric, i.e., does not involve $E$ and $v$, is the main contribution of this paper. We thus gain a new form of the microplane model in which the basic material behavior is not defined as a set of laws for microplane stresses $[(10)]$ but as a set of laws for the evolution of the microplane damage variables $\alpha_{V}, \alpha_{F}$. and $\alpha_{T}$. Then, the macroscopic damage tensor $\alpha_{i, k, m}$ is obtained by integration of the microplane damage variables over the hemisphere using (17). 


\section{Rheology-Independent Derivation of Geometric Damage Tensor}

The foregoing derivation of the damage tensor started from a particular version of the microplane model. At least, the following assumptions were implicitly present in the derivation: (1) The microplane laws are of the form $\sigma=\alpha E^{\prime} \varepsilon[(11)$ and (13)]; (2) the macroscopic stress-strain relationship $\sigma=\alpha E \varepsilon[(11)$ and $(13)] ;$ in of the same type $[(5)]$. involving linear elasticity in its definition $[(15)]$;
is of (3) the initial moduli at microplane level are related to the elastic parameters $E, v,[$ by (14) and (16)]; and (4) the shear stresses and strains on a microplane are parallel $[(10)]$. No references to these hypotheses remain in the final expression in (17), which depends on geometric dimensionless quantities only. Thus, from a practical point of view it is obvious that (17) can be used as a damage model in combination with any rheologic model in the genera context described in the section headed "Effective Stress and Damage Ten sor Uncoupled from Rheology." However, from a theoretical viewpoint the question remains whether the damage tensor derived under these restrictive assumptions could also have been derived using any alternative companion rheologic model or the derivation is exclusive for this case.

Let us start a rheology-free derivation of $\alpha_{i j k m}$ from the assumed basic micro-macro kinematic and equilibrium relations in (6) and (9), which are both rheology independent. On the microplane level we may define the effective (true) stresses, $\tau_{V}, \tau_{b}$, and $\tau_{r}$ such that

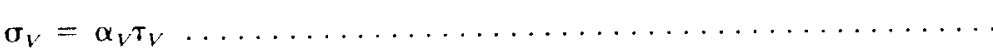

$$
\begin{aligned}
& \sigma_{D}=\alpha_{D} \tau_{D} \\
& \sigma_{T r}=\alpha_{T} \tau_{T}
\end{aligned}
$$

This may be also regarded as a rheology-free definition of damage at microplane level, alternative to (13).

Having assumed the kinematic micro-macro constraint and introduced general microplane stress-strain laws, the microplane stresses cannot (except by chance) represent the resolved components of any stress (macrostress) tensor. The microplane effective stresses in general need not represent the resolved components of any effective stress tensor, i.e., no such tensor needs to exist. However, for a certain special form of the damage ratio tensor, this could occur. Such a property would bring about considerable simplification. To explore it let us introduce the hypothesis that the microplane effective stress components are in fact such resolved components of an effective stress tensor at the macroscopic level, $\tau_{i j}$. This means that, in analogy to (6) for strains, we can write

$\tau_{v}=\frac{\tau_{k k}}{3}=\frac{\delta_{k m}}{3} \tau_{k m}$

$\tau_{D}=\left(n_{k} n_{m}-\frac{\delta_{k m}}{3}\right) \tau_{k m}$

$\tau_{T_{i}}=\frac{1}{2}\left(\delta_{i k} n_{m}+\delta_{i m} n_{k}-2 n_{i} n_{k} n_{m}\right) \tau_{k \prime \prime}$

Let us now substitute (18) and (19) into the right-hand side of (9), and (4) into the left-hand side. After some rearrangements we can factor out $T_{p q}$

$$
\begin{aligned}
& \left\{\frac{\alpha_{V}}{3} \delta_{i j} \delta_{p q}+\frac{3}{2 \pi} \int_{\Omega} \alpha_{p} n_{i} n_{j}\left(n_{p} n_{q}-\frac{\delta_{p q}}{3}\right) \mathrm{d} \Omega+\frac{3}{2 \pi} \int_{\Omega} \frac{\alpha_{I}}{2}\left(n_{i} n_{p} \delta_{i q}+\right.\right. \\
& \left.\left.n_{i} n_{q} \delta_{j p}+n_{j} n_{p} \delta_{i q}+n_{j} n_{q} \delta_{i p}-4 n_{i} n_{j} n_{p} n_{q}\right) \mathrm{~d} \Omega-\alpha_{i j p q}\right\} \tau_{p q q}=0 \ldots
\end{aligned}
$$

This equation should hold for any tensor $\tau_{p q}$. This is possible if and only if the expression within braces, \{\} , vanishes. From this condition we obtain the expression for $\alpha_{i j j_{j},}$ already given in (17). Furthermore, if (19) were violated, it would not be possible to factor out $\tau$ in (20), and (17) would not result. Therefore, the static constraint in (19) is not only sufficient but also necessary to achicve uncoupling of damage from rheology, the latter being defined by the dependence of $\tau_{k}, \tau_{1}$, and $\tau_{T_{i}}$ on the microplane strains. It must be emphasized that this second derivation was not made with recourse to any particular rheologic model, but only on the basis of geometric, kinematic, and equilibrium considerations. This demonstrates from a theoretical viewpoint the general validity of the proposed geometric damage tensor.

Note also that the model represented by the new damage tensor $[(17)]$ coupled with a particular rheologic model is equivalent to a microplane formulation in which there is not only a kinematic constraint for strains but also a static constraint for a certain kind of stresses, namely, the effective stresses. This is a more appealing formulation than that in which there is only a kinematic constraint. The kinematic constraint is reminiscent of parallel coupling of elements in a rheologic model; the static constraint is reminiscent of series coupling. From the modeling of composite materials it is known that the parallel coupling models give the stiffest possible response; whereas the series coupling models give the softest possible response (an example for elastic composites are the Voigt and Reuss bounds). The real behavior can usually be best described by some combination of both types of coupling. It thus appears satisfactory that the foregoing formulation combines both kinematic and static constraints.

\section{Numerical Implementation and Demonstration for Concrete}

Previous studies (Bažant and Prat 1988; Carol et al. 1990) showed that a good and remarkably broad description of the existing test data on the constitutive properties of concrete, encompassing multidirectional tensile strain-softening due to cracking as well as nonlinear triaxial response in the hardening and strain-softening ranges. could be achieved by the microplane model. In those studies all the micromechanics phenomena involved in the microplane laws for $\sigma_{v}, \sigma_{D}$, and $\sigma_{r}$ were mixed. By contrast, in the new model a clear separation has been established between damage effects, represented through the new damage tensor, and rheology, represented by a companion model that can be simple since it does not represent damage (or strain-softening). In this new scheme a separation of tasks can be established between both parts of the model, each characterizing different aspects of the material behavior observed in tests

In the first stage of development presented here, attention is focused on the new microplane damage tensor, and the companion rheologic model is chosen as the simplest possible, i.e. linear clasticity. Choosing a more sophisticated rheologic model, e.g. . elastoplasic, would no doubt broaden the possibilities of representing complex material behavior. 
The evolution laws for microplane damage variables were established on the basis of the laws used for $\sigma_{V}, \sigma_{D}$, and $\sigma_{T}$ by Carol et al. (1990); they were of the form $\sigma(\varepsilon)=\alpha(\varepsilon) E^{0} \varepsilon$. Eliminating from these expressions the factor $E^{0} \varepsilon$, we obtain the equation $\alpha=\exp \left[-(\varepsilon / a)^{p}\right]$, which gives for $\alpha$ a smooth variation from 1 to 0 when the strain increases monotonically; see Fig. 3 for the effect of different values of $p$.

Fig. 4 depicts the general laws for $\alpha_{V}, \alpha_{D}$, and $\alpha_{T}$. In the case of $\alpha_{V}$, as seen in this figure, the basic behavior just described is used only for tension (positive $\varepsilon_{V}$ ), with parameters $a_{1}$ and $p_{1}$ and state parameter $\varepsilon_{V}^{\min }$, which depends on the history. No damage is assumed to occur in hydrostatic compression, where we always have $\alpha_{V}=1$. This is because the volumetric behavior observed in compression tests shows no stress peak and exhibits unloading-reloading branches with almost the initial slope [see Bažant and Prat (1988)], which seems to be modeled by the companion rheologic model of plasticity type better than by the damage tensor itself. If unloadingreloading occurs in tension, the curve given by (2) acts as an envelope, and the maximum strain reached so far is the state parameter depending on history. A jump is assumed for $\alpha_{v}$ if $\varepsilon_{v}$ becomes positive after some damage occurred in tension or vice versa. This kind of jump (and the same kind of jump occuring for $\alpha_{D}$ ) can represent in some way the effect of a straincontrolled microcrack opening and closing at microplane level.

In the case of $\alpha_{D}$, two independent sets of material parameters and state parameters depending on history are used for tension and compression: $a_{1}$, $p_{1}$ (both the same as used for $\alpha_{V}$ ), $\varepsilon_{D}^{\min }$ for tension; and $a_{2}, p_{2}, \varepsilon_{l}^{\max }$ for compression. A jump can also occur for $\alpha_{D}$ when crossing from tensile to compressive parts of the diagram. Finally, $a_{3}, p_{3}$, and $\gamma^{\max }$ are used for the tangential (shear) law, which only shows the positive side since $\gamma$ is the magnitude of the tangential (shear) strain vector. In contrast to Bažant and Prat (1988) and Carol et al. (1990), no dependence of the tangential behavior on any volumetric variable is assumed. This is an aspect of behavior directly caused by internal friction rather than by damage itself, and therefore migh be better handled by the companion rheologic model.

The model was implemented in a computer code in the manner described by Carol et al. (1990), with 28 spatial orientations of microplanes (Bažan and $\mathrm{Oh}$ 1985) for which history is recorded and damage evaluated. The

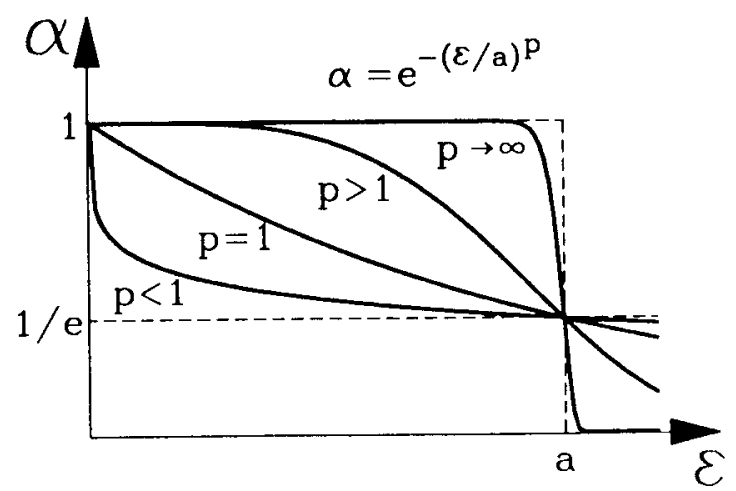

FIG. 3. Basic Exponential Curve for Damage Evolution
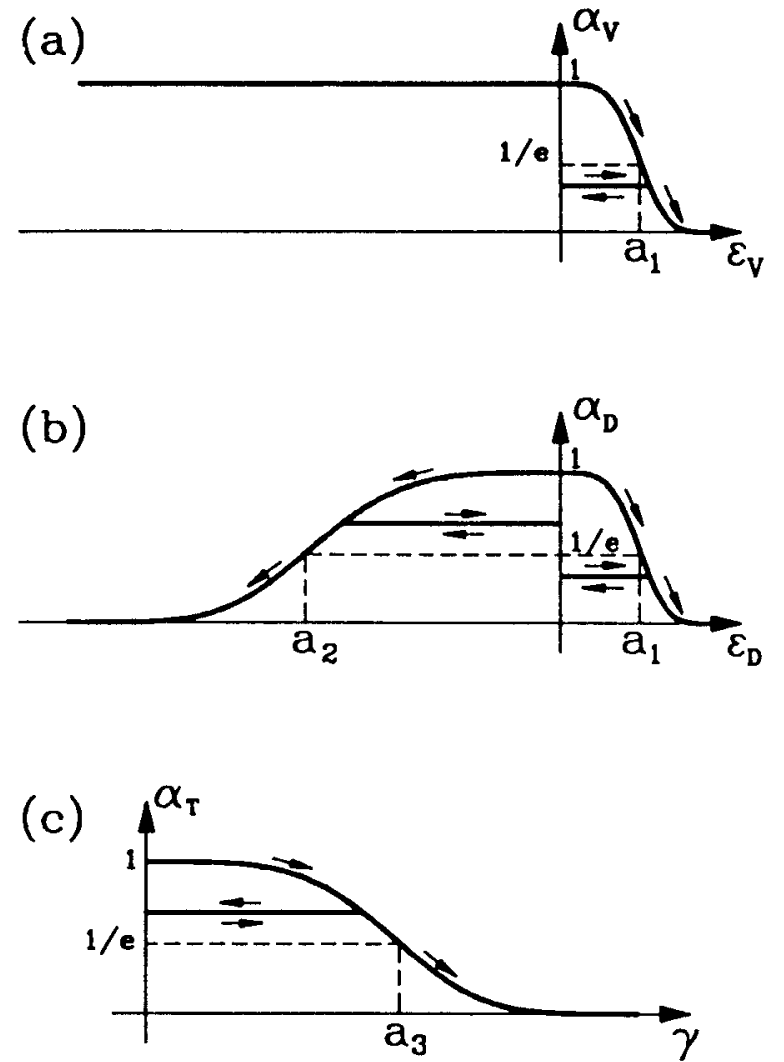

FIG. 4. Microplane Damage Laws: (a) Volumetric Damage $\alpha_{v}$; (b) Normal Deviatoric Damage $\alpha_{1}$; (c) Tangential (shear) Damage $\alpha_{T}$

stresses are computed explicitly from specified strain increments of finite size, and subroutines co-opted from a "single-point" or "full-finite element (FE)" computer program are also used. In fact, no major differences with regard to the previous version of microplane model exist from this viewpont except that the laws are established in ternis of microplane damage variables instead of microplane stresses and that, after the damage tensor has been obtained, additional steps are needed to obtain the new values of $\tau_{i j}$ and to evaluate the corresponding tensorial product in (4) that gives the new $\sigma_{i}$

There are six material parameters of the model for the microplane damage tensor: $a_{1}, p_{1}, a_{2}, p_{2}, a_{3}$, and $p_{3}$. However, the values $p_{1}=0.5 ; p,=1.5$ and $p_{3}=1.5$ can be assigned for most of practical cases, thus reducing the number of material parameters to be determined from test results to only three. The number of history-dependent state variables for 28 microplane directions is 85 (one for the volumetric curve. same for all microplanes; two for the deviatoric curve; and one for the shear curve on each microplane). Furthermore, the material parameters of the rheologic model ( $E$ and $v$ in this case) and possibly its internal variables (none in this case) may also be counted. 
As an example, a uniaxial test reported by van Mier in 1984, in which both longitudinal stress and transverse strains were measured, is reproduced with the model. The parameters used to fit these data are: $E=2,406 \mathrm{MPa}$; $\nu=0.18 ; a_{1}=0.0004 ; a_{2}=0.006 ;$ and $a_{3}=0.0018$. The remaining parameters have their general values already given. The results are represented in Fig. 5 by solid lines. The dots are the experimental data and the dashed lines are the results obtained with the previous version of microplane model for the same example. It can be observed that both curves are very similar in each diagram, which should not be surprising since both models are almost equivalent in this example, in which no volumetric dependence
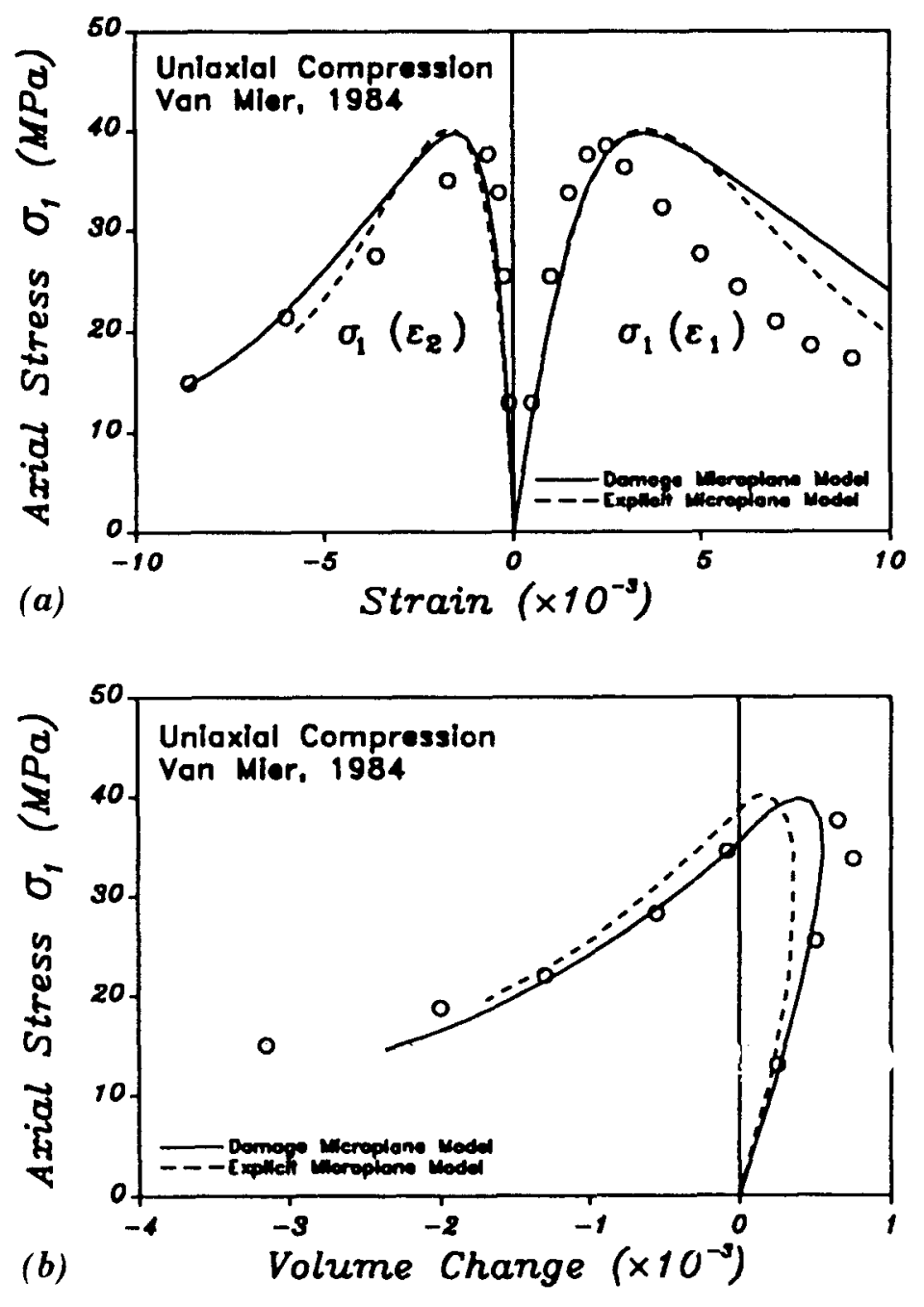

FIG. 5. Comparison with Unlaxial Test by van Mier (1984) was assumed for $\sigma_{r}$ in the computations with the previous microplane model (Carol et al. 1990). The only difference arises from the parameter $\eta_{0}$, which existed in the old model but now has disappeared from the formulation. In the section headed "Derivation of Geometric Damage Tensor from Concrete Model" it was shown that the full equivalence between both models occurs for $\eta_{1}=(1-2 v) /(1+v)$ in the old model. For $v=0.18$ this means about $\eta_{0}=0.54$; the value taken in the previous computations for this example was 0.85 . Due to that difference, the models are not completely equivalent, and the parameters $a_{1}, a_{2}$, and $a_{3}$ cannot have the same values. It is clearly shown in the example, however, that the restriction over the value of $\eta_{0}$ does not prevent the model from yielding a very good fit of experimental data. This is not surprising, since Bažant and Prat (1988) found the effect of $\eta_{0}$ in the closeness of fit to be minor and that the optimized $\eta_{0}$-values were mostly quite close to those from (16). Finally we must emphasize that Fig. 5 does not represent an experimental verification of the present model. Rather, this verification rests on the fact that: (1) The Predictions of the present and previous models are quite close; while (2) the experimental verification of the previous model was extensive.

\section{CONCLUSIONS ANo BRoader IMPLICATIONS}

For the general microplane model characterized by a kinematic micromacro constraint it is possible to formulate a fourth-order damage tensor in a form that is uncoupled from the rheologic constitutive properties of the material. In this formulation the damage is solely of geometric origin, being due to the reductions of the net stress-carrying cross-sectional area fractions for various orientations in the microstructure. The damage tensor characterizes the values of these area fractions for all spatial directions, and permits them to have any values. The damage tensor formulated in this manner fits well the framework of continuum damage mechanics.

Despite the assumed kinematic constraint between macrostrains and microstrains, it is possible to satisfy at the same time and additional static constraint such that the effective microstresses on the microplanes are the resolved components of the effective macrostresses.

The fact that the constitutive model is composed of two independent parts (one for damage, another for rheology) allows better insight, facilitates the fitting of test data, and increases versatility.

A recent microplane model for tensile and compressive strain softening and general nonlinear triaxial behavior of concrete, which has been amply verified by test data, can be easily adapted to the geometric damage form without any appreciable detriment to the closeness of test data fits.

The possibility of uncoupling geometric damage from constitutive (rheologic) properties is attractive for development of more general constitutive models combining damage with creep and rate effects, with complex unloading and reloading paths, and with cyclic loading. It might be possible to model such behavior adequately using some existing plastic, viscoelastic, viscoplastic, or nonlinear cyclic material model without damage, and then incorporating into it the geometric damage tensor deduced here (17)]. By separating damage, the constitutive (rheologic) model, which relates here the effective (microscopic, true) stress tensor (instead of the macroscopic stress tensor) to the strain tensor, can probably be much simpler than it would have to be if the damage were mixed with nondamage constitutive properties. By virtue of the geometric damage concept constitutive modeling 
can be restricted to the material between the microcracks and voids in the microstructure leaving the overall effect of the growth of the microcracks and voids as the only behavior to be described by the geometric damage tensor.

\section{ACKNOWLEDGMENTS}

Partial financial support was obtained under NSF grants BCS-8818230 and AFOSR grant 91-0140 to Northwestern University in the early and later phases of research. Partial support for related studies of micromechanics of damage were received from the NSF Center for Science and Technology of Advanced Cement-Based Materials (ACBM), at Northwestern University. The first author is grateful for financial support under a NATO fellowship. and the third author is grateful for a fellowship from the Ministry of Education of Spain. Partial support from the Spanish CICYT under research projects PA85-(0321 and PB87-0861 is also gratefully acknowledged.

\section{Appendix 1. References}

Aboudi, J. (1987). "Constitutive relations for cracked metal matrix composites." Mech. Mater. 6, 303-315.

Attiogbe, E. K., and Darwin, D. (1987). "Self consistent model for transversely isotropic cracked solid." J. Engrg. Mech., ASCE, 113(7), 984-999.

Batdorf, S. B., and Budiansky, B. (1949). "A mathematical theory of plasticity based on the concept of slip." Tech. Note 1871, National Advisory Committee for Aer onautics (NACA), Washington, D.C

Bažant, Z. P. (1984). "Microplane model for strain-controlled inelastic behavior." Mechanics of engineering materials, C. S. Desai et al., eds., John Wiley, Chichester, NY, 45-59

Bažant, Z. P., and Carol, I. (1990). "Geometric damage tensor, uncoupled from constitutive properties, and current status of strain-softening models." Developconstitutive properties, and current status of strain-softening models." Develop-
ments in theoretical and applied mechanics: Proc., 15th Southeastern Conf. on ments in theoretical and applied mechanics: Proc., 15th Southeastern Conf. on
Theoretical and Appl. Mech., Vol. 15, S. V. Hanagut et al., eds., Georgia Institute of Technology. Atlanta, Ga. $927-933$.

Bažant, Z. P., and Cedolin, L. (1979). "Blunt crack band propagation in finite element analysis." J. Engrg. Mech. ASCE, 105(2), 297-315.

Bažant Z P and Chern, J.C. (1985). "Strain-softening with creep and exponential algorithm."J. Engrg. Mech., ASCE, 111(3), 559-582.

ažant, Z.P. and Gambarova, P. G. (1984). "Crack shear in concrete: Crack band microplane model." J. Struct. Engrg., ASCE, 110(9), 2015-2035.

Bažant, Z. P.. and Kim. S. S. (1979). "Plastic-fracturing theory for concrete." $J$ Engrg. Mech., ASCE, 105(3), 403-428

Bažant, Z. P., and Oh, B. H. (1983). "Crack band theory for fracture of concrete." Mat. Struct., 16, 155-177.

Bažant,.Z. P., and Oh, B. H. (1985). "Microplane model for progressive fracture of concrete and rock." J. Engrg. Mech., ASCE, 111(4), 559-582.

Bažant Z. P. and Prat, P. C. (1988). "Microplane model for brittle-plastic material I: Theory." J. Engrg. Mech., ASCE, 114(10), 1672-1702.

Bažant, Z P., and Prat, P. C. (1988). "Microplane model for brittle-plastic material. II: Verification." J. Engrg. Mech., ASCE, 114(10), 1672-1702

Betten, J. (1983). "Damage tensors in continuum mechanics." J. Mécanique Théorique et Appliquée, $2(1), 13-32$

de Borst, R. (1987). "Computation of post-bifurcation and post-failure behavior of strain-softening solids." Computers and Struct., 25(2), 211-224.

Budiansky, B., and O'Connell, R. J. (1976). "Elastic moduli of a cracked solid." Int. J. of Solids and Struct., 12.81-97.

Carol, I. (1989). "Explicit models for non linear time-dependent behavior of concrete in compression hased on damage concepts and the aging Maxwell chain. ". Ird Jount ASCE/ASME Mech Conf. ASCE. New York, N.Y.

Carol, L. Bažant, Z. P. and Prat, P. C. (1990). "New explicit microplane model for concrete: Theoretical aspects and unified implementation for constitutive verification and finite element analysis." Report (3T015/1990), ETSECCPB-UPC. Campus Nord, Edif. D2. Gran Capità, s/n. E-(08034 Barcelona, Spain.

Carol, 1., and Prat, P. C. (1990). "A statically constrained microplane formulation for the smeared analysis of concrete cracking." Proc., 2nd. Int. Conf. on ComputerAided Analysis and Design of Concrete Sirut., Zell Am See, Austria, Vol. 2, 919 930.

Chaboche, J. L. (1978). "Description thermodynamique et phénoménologique de la viscoplasticité cyclique avec endommagement" (in French), thesis, presented to the University of Paris IV, at Paris, France, in partial fulfillment of the require ments for the degree of Doctor of Philosophy

Chaboche I (1979). "The concept of effective stress applied to elasticity and viscoplasticity in the presence of anisotropic damage." ONERA Report 1979/77. Chaboche, J. L. (1981). "Continuum damage mechanics: A tool to describe pheChaboche, J. L. (1981). "Continuum damage mechanics: A tool to describe
nomena before crack initiation." Nuclear Lingrg. and Design 64. 233-247.

Costin, L. S. (1985). "Damage mechanics in the post-failure regime." Mech. of Mat, , 4, 149-160.

Costin, L. S., and Stone, C. M. (1987). "Implementation of a finite element damage model for rock Constitutwe law' for cngineering materials: Theory and Applications, C. S. Desai et al., eds. Elsevier. New York. N.Y.

Cowin, S. C. (1985). "The relationship between the elasticity tensor and the fabric tensor." Mech. of Mat., 4, 137-147.

Crisfield, M. A. (1982). "Local instabilities in the non linear analysis of reinforced concrete beams and slabs." Proc., Inst. of C11. Engrg. . 73, 135-145.

Davidson, L., and Stevens, A. L. (1973). "Thermomechanical constitution of spalling elastic bodies." J. Appl. Phys. 44,667-674.

Dougill, J. W. (1976). "On stable progressively fracturing solids." J. of Appl. Math ematics and Physics (ZAMP). Basel, Switzerland, 27, 42.3-436.

Dragon, A., and Mróz, Z. (1979). "A continuum model for plastic-brittle behavior of rock and concrete." Int. J. Engrg. Sci., 17, 121-137.

Franziskonis, (B.. and Desai, C. S. (1987) . "Constitutive model with strain softening." Int. J. of Solids and Struct., 23, 733-750.

Hillerborg. A. (1984). "Numerical methods to simulate softening and fracture of concrete." Fracture mechanics applied to concrete structures, G. C. Sih, ed., Martinus Nijhoff. The Netherlands.

Hillerborg, A., Modéer, M., and Pettersson. P. E. (1976). "Analysis of crack formation and crack growth in concrete by means of fracture mechanics and finite elements." Cement and Concrete Res. 6(6), 773-782.

Horii, H., and Nemat-Nasser. S. (1983). "Overall moduli of solids with microcracks: Load-induced anisotropy." J. of Mech. and Physics of Solids. 31(2). 155-171.

Kachanov, L. M. (1958). "Time rupture process under creep conditions" (in Russian). Izv' Akad Nauk SSR. Otd Tekh Nauk 8, 26-31.

Kachanov, M. (1980). "Continuum model of medium with cracks." J. Engrg. Mech. ASCE $106(5), 1039-1051$

Kachanov. M. (1987). "Elastic solius with many cracks: A simple nurhod of analysis." Int. J. of Solids and struct., 23(1), 23-43.

Krajcinovic, D., and Fonseka, G. U. (1981). "The continuous damage theory of brittle materials." J. of Appl. Mech. . 48. 809-824

Leckie, F. A. (1978). "The constitutive equations of continuum creep damage mechanics." Phil. Trans. of the Roval Society. London, Engtand, 288. 27-47.

Leckie, F. A.. and Onat, E. T. (1980). "Tensorial nature of damage measuring internan varibles." Proc, IUTAM Symp., Senlis. France.

Lemaitre, J. (1984). "How to use damage mechanics." Nuclear Engrg. and Design, $80,233-245$.

Lemaitre, J., and Chaboche, J. L. (1978). "Aspect phénoménologique de la rupture par endommagement" (in French), J. de Mécanique Appliquée, 2(3), 317-365. 
Lemaitre, J.. and Chaboche, J. L. (1985). Mécanique des materiaux solides (in French), Dunod, Paris

Lin, T. M. (1968). Theory of inelastic structures. John Wiley and Sons Inc., New York, N.Y

Mazars, J. (1985). "A model for a unilateral elastic damageable material and its application to concrete." Proc., RILEM Int. Congress on Fracture Mech. of Concrete, Lausanne, Switzerland.

van Mier, J. G. M. (1984). "Strain-softening of concrete under multiaxial loading conditions," thesis presented to the University of Eindhoven, at The Netherlands. in partial fulfillment of the requirements for the degree of Doctor of Philosophy.

Murakami, S. (1987). "Anisotropic damage theory and its application to creep crack growth analysis." Constitutive laws for engineering materials: Theory and applications, C. S. Desai et al., eds, Elsevier, New York, N.Y., 187-194.

Murakami, S and Ohno, N (1980). "A continuum theory of creep and creep damage. " Creep in Structures. A. R. S. Ponter et al., eds., Springer Verlag, Berlin. Germany, 422- 444

Nemat-Nasser, S., Iwakuma, T., and Hejazi, M. (1982). "On composites with periodic structure." Mech. of Mat. $1,239-267$.

Oda, M. (1983). "A method for evaluating the effect of crack geometry on the mechanical behavior of cracked rock masses." Mech. of Mat., 2, 163-171.

Ortiz, M. (1985). "A constitutive theory for the inelastic behavior of concrete." Mech. of Mat., 4, 67-93.

Ortiz, M. (1987). "An analytical study of the localized failure modes of concrete." Mech. of Mat., 6, 159-174.

Ortiz, M., and Quigley, J. J. (1989). "Element design and adaptative meshing in strain localization problems." Computational Plasticity, D. R. J. Owen et al., eds. Pineridge Press, Swansea, Wales.

Pijaudier-Cabot, G., and Bažant, Z. P. (1987). "Non-local damage theory." J. Engrg. Mech., ASCE, 113(10), 1512-1533.

Rabotnov, Y. N. (1969a). Creep problems of structural members. North-Holland, Amsterdam, The Netherlands.

Rabotnov, Y. N. (1969b). "Creep rupture." Appl. Mech. Conf., Heteny et al., eds. Stanford University, Stanford, Calif., 342-349.

Resende, L. (1987). "A damage mechanics constitutive theory for the inelastic behesende, L. (1987). "A damage mechanics constitutive theory for the inelastic be-

Simó, J. C., Ju, J. W., Taylor, R. L., and Pister, K. S. (1987). "On strain-based continuum damage models: Formulation and computational aspects." Constitutive laws for engineering materials: Theory and applications, C. S. Desai et al., eds., Elsevier. New York, N.Y., 233-245.

Suaris, W. (1987). "A damage theory for concrete incorporating crack growth characteristics." Constitutive laws for engineering materials: theory and applications. C. S. Desai et al., eds., Elsevier, New York, N.Y.

Suaris, W., and Shah, S. P. (1984). "Rate-sensitive damage theory for brittle solids." J. Engrg. Mech., ASCE, $110(6), 985-997$.

Taylor, G. I. (1938). "Plastic strain in metals." J. Inst. Metals, 62, 307-324.

Vakulenko, A. A., and Kachanov, M. L. (1971). "Continuum theory of cracked media" (in Russian). Mekh Tverdogo Tela. U.S.S.R., 6, 159-166.

Yazdani, S., and Schreyer, H. L. (1988). "An anisotropic damage model with dilatation for concrete" Mech of Mat., 7, 231-244. 